\title{
Off-line synchronization of measurements based on a common pseudorandom binary signal
}

\author{
PIET J. HOOGEBOOM \\ National Aerospace Laboratory NLR, Amsterdam, The Netherlands
}

\begin{abstract}
Postprocessing (or off line) synchronization methods rely on some overlap of information between two or more simultaneous measurements. The methods vary in the amount of explicit data needed to record the overlap: on the one hand, the full recording of an (absolute) time source for each measurement or, on the other hand, the reliance on information overlap of different parameters in the measurements. Whenever it is impossible to record the time from a central clock in all the simultaneous measurements, it is often possible to record a simple common signal. Using a binary pseudorandom noise (PRN) code to modulate the common signal enhances extraction of relative time information. The simplicity of this binary code makes it suitable for recording on various media (digitally or within audio and video streams) without the requirement for specialized and/or complex converters. The PRN technique has been applied successfully by NLR in several projects executed in a range of environments over the last decade and has been shown to provide time difference information with high precision.
\end{abstract}

The recording of data on a single recorder is often used as a method by which to preserve the time relations between the various measured signals. The advent of modern computers allows storing of data in separate data files using information from a common clock, giving more freedom in data storage while maintaining the required time relations. For ambulatory experiments, however, it is often necessary to record data with separate devices without the availability of overlapping clock information. In those cases, it is often possible to record a simple common signal at all recording places that can be used later for synchronization. The signal used can be diveres (e.g., electrical, sound, or light). For example, when recording video and performance data, one can use a flashing (infrared) lightemitting diode (LED). The flashing sequence can be recorded electronically with the performance data, whereas the (ambient) light changes can later be recovered from the video information. This article provides a solution for the commonly encountered problem of how to determine time differences between simultaneous measurements, using (pieces of) overlapping information.

An example of a generic set-up of a measurement system with multiple recorders (and thus, the data synchronization problem) is given in Figure 1. Other examples of measurement systems with similar synchronization problems are reported in, for instance, Harlaar, Redmeijer, Tump, Peters, and Hautus (2000), and Geuze and Hunnius (2002).

The synchronization problem is illustrated in Figure 2, which shows two separate measurements, 1 and 2, each

Correspondence concerning this article should be addressed to P. J. Hoogeboom, National Aerospace Laboratory NLR, Flight Division, P. O. Box 90502, 1006 BM Amsterdam, The Netherlands (e-mail: hoog@nlr.nl). containing multiple recorded channels (parameters $1 \ldots m$ and $1 \ldots n$, respectively).

For the synchronization, the following need to be achieved.

1. Correction of the start time of each measurement. Variations are caused by the impossibility of starting all recordings at exactly the same time. So the measurements need to be shifted in time in order to match the location of the recorded start pulse.

2. Compensation for differences in recording speeds. This is shown in the figure as a different interval between the start and the stop markers. Several causes can be mentioned for such a difference. The simplest one is a difference in recorder clock speed (on the basis of practical experience, we can state that clock speed differences are normally less than approximately $0.2 \%$; Stein \& Vig, 1992; Vig, 2001).

3. Resolving measurement gaps or frame drops; these occur when data records are missed in the recording process.

\section{Synchronization Methods}

If it is assumed that no measurement gaps are present, the simplest solution is to shift the second measurement in time to match the start of the first measurement, followed by stretching or compressing the second measurement to also match the end of the first measurement. In other words, shift and stretch to match, or mathematically, apply a time offset and a time gain. The actual time of a given value of a parameter from measurement $Y$ is then given by

$$
\begin{aligned}
T_{p y} & =T_{\text {measurement } Y}+T_{\text {offset } Y} \\
& +\left(T_{\text {parameter }}+T_{p \_ \text {offset }}\right) * \text { Time_gain }_{Y},
\end{aligned}
$$

where $T_{p y}$ is the time of parameter $p$ of measurement $Y$, $T_{\text {measurement } Y}$ is the start time of measurement $Y, T_{\text {offset } Y}$ is 


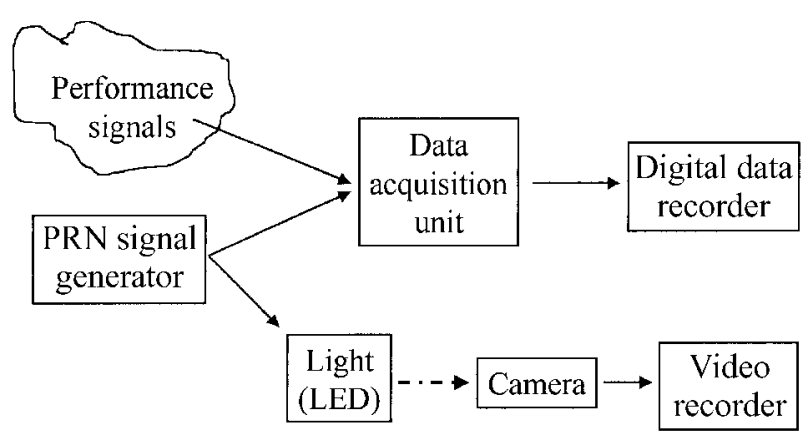

Figure 1. Example measurement set-up with a data synchronization problem.

the offset in the start time of measurement $Y$ (relative to the reference measurement), $T_{\text {parameter }}$ is the time of the parameter value relative to the start of the measurement, $T_{p_{-} \text {offset }}$ is the optional offset specific for parameter $P$ (relative to the other parameters in the same measurement; in most cases, this value is assumed to be equal to zero and, therefore, will be further ignored in this article), and Time_gain ${ }_{Y}$ is the time gain for measurement $Y$.

In order to synchronize multiple measurements off line, some overlapping common information needs to be present in each measurement. The simplest one is to record a unique event, such as a start-marker. This start-marker is subsequently used to shift the measurements in time. Unfortunately, it provides only a time offset and no time gain. As a consequence, longer intervals of measurements will reduce the accuracy progressively. A first extension of the start-marker method is the addition of an end-marker. Using two markers, well separated in time, allows for a determination of the time gain. This two-marker method has advantages in case one of the markers was not recorded but is limited by the accuracy of the measurement of the marker information. For example, the signal conditioning (such as amplification and filtering) and sampling frequency determine the obtainable reconstruction and timing accuracy of the marker signals. If the required time resolution is more demanding, the approach of using just one or two markers may be insufficient, and time has to be recorded in a more continuous way.

\section{Continuous Time Recording}

The need for synchronization requires that (absolute) time can be reconstructed in all simultaneous measurements. If data of different modalities are to be recorded (e.g., performance data and video), some translation of the time information to the other modalities may be required. For video media, use can be made of the vertical interval time code (VITC) method, which allows recording of digital information in hidden or invisible video lines. Those hidden lines are not converted to computer-based video formats (unless a work-around is provided for), making the VITC method often less suitable. An alternative is to record time information in the visible part of the video signal, since this survives the conversion to digital video for- mats. However, the difficulty is that one has to retrieve the recorded time information in a computer-readable format. Another drawback is the limited video resolution.

The audio channel can also be used for storing timerelated information. The bandwidth of the audio channel is limited, resulting in restrictions for the stored amount of information. Also the time relation between video and audio is guaranteed only for some hundreds of milliseconds, since the design driver for the conversion soft- and hardware is lip-synchronic replay. So, although feasible, techniques utilizing the audio channel are highly dependent on the hardware set-up used and its quality for the digitization of the analogue information. The rest of this article focuses on a practical method that does not require much space within the video image and that can also be ported to the audio domain or to other digital and analogue recording systems.

\section{Use of Information Overlap}

Measurements can be synchronized by maximizing the similarity of common information at each point in time. To this end, three different interactive synchronization methods have been tested in the NLR HEART data analysis package (Hoogeboom, 2000).

1. Trial and error. The user is given the option to numerically enter the time offset and time gain values of a measurement, after which the data are updated to reflect the changes (e.g., time traces of the signals to be matched).

2. Graphical slewing of measurements. This method allows the user to select a characteristic point in one of the measurements, followed by a similar point in the other measurement. Subsequently, the time difference between the points is used to update the time offset.

3. Graphical matching of four points. Two reference points are compared with two others in the measurement to be synchronized. This method allows the determination of the time offset and the time gain.



Figure 2. Two simultaneous measurements, each with a start and a stop pulse. For the synchronization, the locations of the start and stop pulses need to be matched by shifting Measurement 2 to the left (time offset) and by stretching it (time gain). 
All three options are powerful and very useful, but the signal synchronization is essentially performed manually. Fortunately, automated synchronization can be achieved by a matching process, corresponding in mathematical terms to maximizing the correlation of the two signals. In this case, the standard correlation value needs to be extended to a correlation function, $C(d t)$, which provides the measure of similarity between two signals with a time offset, $d t$ :

$$
C(d t)=\frac{\sum x(t) * y(t-d t)}{\sqrt{\sum x(t)^{2} * \sum y(t-d t)^{2}}} .
$$

Normally, the function is computed over a certain window length $(-N \ldots N)$, as is implied by the sigma function. Also, it is assumed that the means of $x(t)$ and $y(t)$ are equal to zero.

In Figure 3, two time traces are plotted. The first (top) one is the height above a reference system, as derived from a global positioning system (GPS). The second channel shows the altitude of the aircraft, as derived from barometric air pressure. Both signals have similar patterns, with the barometric altitude signal slightly leading the GPS height signal. The third trace shows the correlation function, $C(d t)$, for the two signals. This function peaks left of the center line (which equals to zero time difference between the signals, or $d t=0 \mathrm{sec}$ ). The time difference between the two signals is determined to be $-13 \mathrm{sec}$, meaning that the altitude is $13 \mathrm{sec}$ in advance of the GPS height channel. The correlation function peak itself is not very sharp and limits the accuracy of the time difference estimation.

\section{Pseudorandom noise}

To enhance the resolution of the correlation function, a special digital signal can be used. The requirements for its use can be summarized as its having a high correlation for signals without time offsets and a low correlation for signals with some time difference. Also, the code repetition distance should be sufficient to avoid inappropriate locks. Substantial research has been performed in the area of pseudorandom noise (PRN) codes (Brown \& Hwang, 1992; Dixon, 1994; Gold, 1967, 1968; Pursley \& Roefs, 1979; Stein \& Vig, 1992). Some navigation systems, such as the GPS (Anonymous, 1997; Lin \& Tsui, 2000) even rely solely on similar types of codes. Also, similar techniques are widely used in cellular phone systems.

Figure 4 shows a typical example of two different measurements containing overlapping PRN information: $P R N$ sequence and shifted PRN sequence. The code generation frequency is $10 \mathrm{~Hz}$. The correlation function, $C(d t)$, of the two signals is indicated in the third trace and peaks about $3 \mathrm{sec}$ before the no-time-offset location (vertical line in the middle of the window). This is confirmed by the two time histories, from which it is easy to see that the shifted PRN sequence is earlier than the original PRN sequence.

The peak in the correlation function is now sharp and also relatively high when one considers a time difference of $3 \mathrm{sec}$ combined with a data window length of only $10 \mathrm{sec}$. Note that increasing the data window length enlarges the peak/noise ratio, but at the cost of prolonged computation times.

The practicality of the PRN-based time difference detection was tested with several data sets in the context of the Visual Lab project (Noldus, 1999). The accuracy of the time difference determination was found to be dependent on the PRN code used, the data window length applied, the minimum time step $(d t)$ used in the correlation function, the method of locating the peak in the correlation function, and the time gain difference between the measurements. The time required for the computation of the correlation function depends on a similar set of parame-
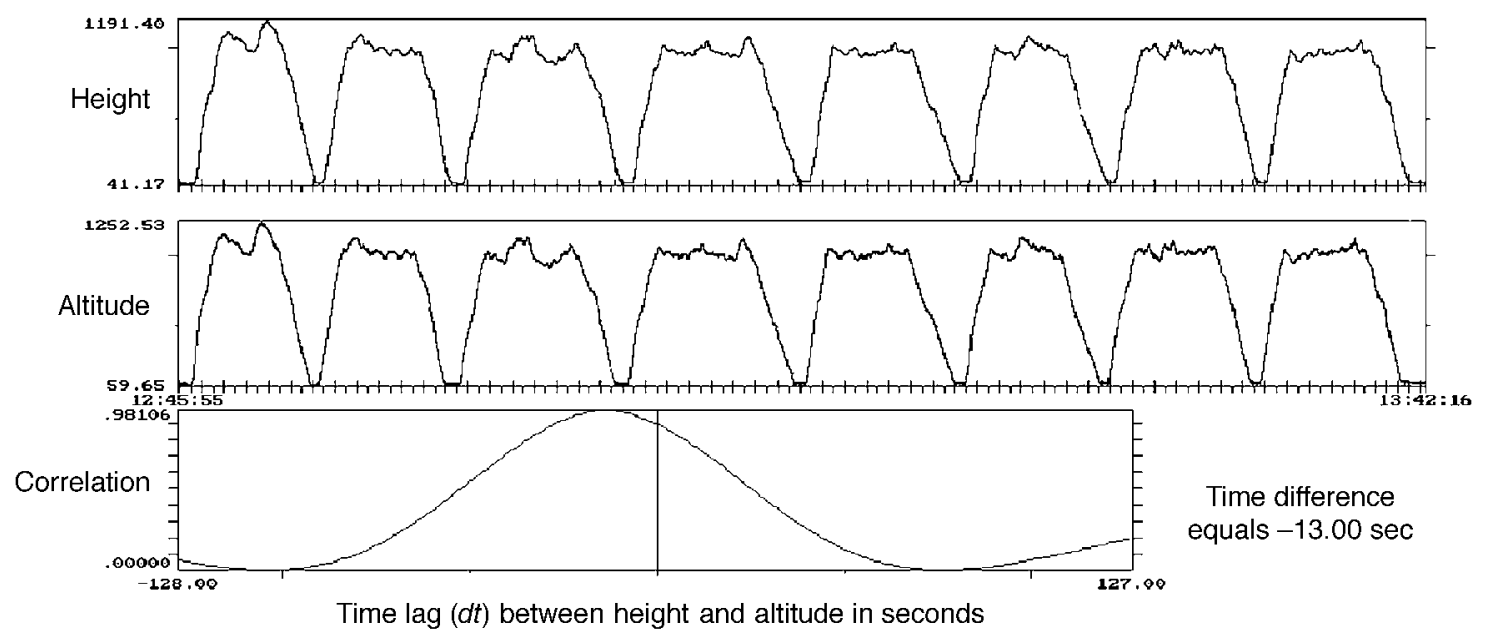

Figure 3. Example correlation function (bottom trace) showing the correlation of two signals (upper two traces) relative to the time offset between those two. The correlation function peaks left of the no-time offset location in the center, indicating that the upper trace is delayed by $13 \mathrm{sec}$, relative to the middle trace. 


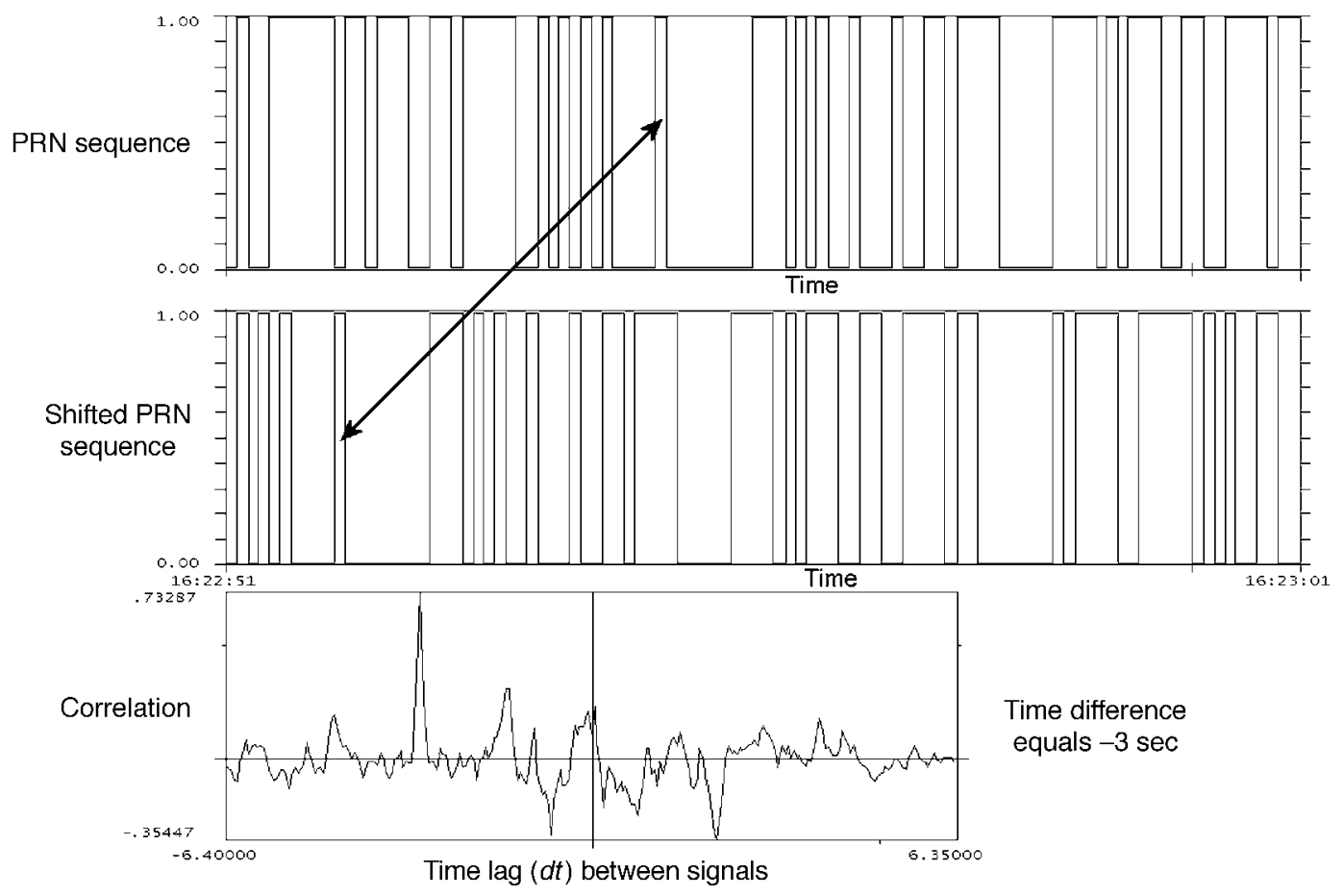

Figure 4. Correlation function of a pseudorandom noise (PRN) signal for an interval of 10 sec and a relative time difference of 3 sec, with the middle trace leading the upper trace.

ters. A higher accuracy requires longer computation times. Therefore, for most applications, a tradeoff exists between the required accuracy and the allowed computation times.

Surprisingly, it was found that major distortions to the PRN signal are allowed: Due to the correlation process, local differences (e.g., small measurement errors) are averaged out and have almost no influence on the accuracy of the determined time difference. This property of the correlation function makes the PRN technology relatively robust, as compared with the use of a single- or a dualmarker technique.

\section{Detection of Measurement Gaps With the PRN Code}

So far, only the linear situation has been looked atmeaning the derivation of the time offset and the time gain, while ignoring the possible presence of missing records. To test the behavior of the PRN in such cases, a test file was created consisting of a continuous PRN sequence and a (deliberately) interrupted PRN sequence.

The top time trace offset in Figure 5 presents the time offset of the second PRN sequence, relative to the first. Each jump indicates that a certain number of records were discarded in the second sequence. The magnitude of the jump indicates the amount of time skipped. The interrupted PRN sequence was stretched by applying a gain difference of $0.5 \%$. Both data sequences were compared, using the correlation technique with a sliding window of 100 clock intervals, stepping at a pace of 10 clock inter- vals. (Note that the clock interval, or chip, is the inverse of the code generation frequency; with a code generation frequency of $10 \mathrm{~Hz}, 10$ intervals correspond to a window step size of $1 \mathrm{sec}$.) The result of the automatically determined time difference is given by the second trace, which jumps at the expected locations. The slope of the second curve is caused by the artificially introduced time gain difference. Stretching the second run compensated for the lack of data records; therefore, the jumps were expected to go in the opposite direction (as compared with the slope caused by the time gain difference). As such, it can be concluded that the PRN technique can (1) detect measurement gaps and (2) provide quantitative information on both the location in time and the magnitude of the gap. The relatively clear detection of the location of the time offset jump can be explained by the mechanism of the correlation algorithm in combination with the applied peak detection algorithm. Close to a time jump, the correlation function, $C(t)$, will show two peaks. One peak belongs to the data segment just before the time jump, whereas the other peak belongs to the data segment after the jump. The magnitudes of the peaks depend on the relative size of the data segments, with the largest peak belonging to the largest data segment within the correlation window. At the moment at which both peaks are of equal magnitude, the size of both data segments is equal, and hence, the time jump is in the middle of the correlation window. Any change in this situation will force the peak detection algorithm to select the highest peak, which causes a jump in the detected time differ- 




Figure 5. Measurement gap detection. The first trace indicates the location and magnitude of artificially introduced record losses between two pseudorandom noise recordings. The second trace indicates the determined time difference between the two recordings, using the correlation-based approach. Both the occurrence and the magnitude of the measurement gap are detected. The slope in the second trace indicates a difference in time gain between the two recordings.

ence. As such, the accuracy of the jump location depends on the accuracy of the peak detection algorithm and on the time increment used in the correlation function.

\section{Practical Recording of $P R N$ in Video Signals}

The practicality of the PRN technique can be illustrated by our experiences gathered in, among others, the DIVA (design of human factors interfaces and their validation in aeronautics) project(Nibbelke, Ferro, \& Hoogeboom, 2001), which was cosponsored by the European Commission. In this project, pilot/crew performance and behavior were measured in a flight simulator. The recorded signals included physiological data and data from the simulator, as well as video observations. In this specific case, security reasons prohibited a direct connection of the recording equipment (consisting of several computers) to the simulator network. In addition, to minimize the risk of electrical shocks for the crew, connecting the physiological recording equipment directly to the simulator computers was (and is) not allowed. Therefore, a simple interface was used through a specially developed break-out box, providing the necessary insulation. The simulation equipment transmitted the digital PRN code through this box to the recording equipment. A green LED allowed recording of the PRN information on one of the four videos taken from the cockpit. In the video stream, the LED image had a size of approximately 15 pixels (horizontally). After combination of the four video streams (resolution reduction by a factor of 2) and conversion to MPEG 1 (another resolution reduction by a factor of 2), only a couple of pixels remained in the final digital image. An example screen shot of the resulting video is given in Figure 6.

The digitized video was subsequently used to extract the PRN information by allowing the data analyst to select an area on the screen. The height and width of the selection square were approximately 3 pixels. The video was replayed in a window with a width of 352 pixels. The total intensity of the square was calculated on the basis of the RGB values of the pixels within the selection square (repeated for each video frame). If the intensity of the square was equal to or above a user-selectable threshold, the status of the LED was assumed to be ON; otherwise, it was set to OFF. The status of the LED was subsequently stored in a data file for further processing. During this experiment, the following lessons were learned.

1. The background of the LED should be dark enough to be able to discriminate between the two states.

2. The use of a stationary scene camera worked well. During each simulator run, the location of the LED in the image was stationary. However, between different runs, the location could change a little, forcing the data analyst to readjust the selected detection area.

3. Sometimes, data were lost due to a person's walking between the camera and the LED. Depending on the selected threshold and the color of the clothes, the LED status was fixed to either the ON or the OFF mode. 


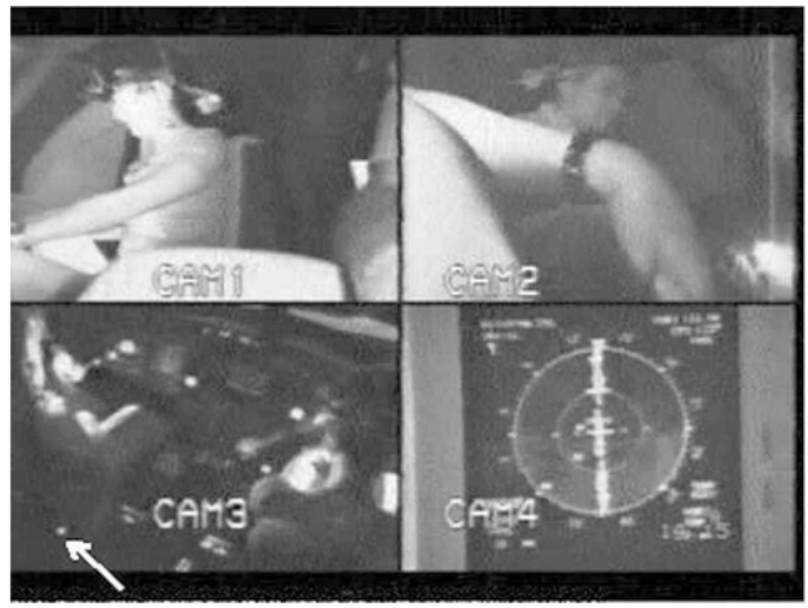

Figure 6. Example video screen shot. The arrow indicates the location of the light-emitting diode.

4. With the small selected size of the detection square, the observed intensity difference was about 80 (on a scale from 0 to 255).

As a whole, it was surprisingly simple to retrieve the code information.

The correlation function of the video-derived PRN signal and the separately measured electrical signal indicated that the video was delayed at the start of the measurement by approximately $2.31 \mathrm{sec}$. At the end of the measurement, the video was leading with $1.4 \mathrm{sec}$. The total time differ- ence was $3.7 \mathrm{sec}$ over a measurement length of $2,244 \mathrm{sec}-$ in other words, a time gain difference of $0.165 \%$.

An alternative approach, utilizing the signal changes to estimate the clock interval from observed data, revealed that the actual code generation frequency was $1.79 \mathrm{~Hz}$. The time gain difference between the two measurements was estimated to be $0.18 \%$, which is close to the value derived above. Note that this alternative method is less precise and is not able to estimate the time difference between the measurements. The main benefit of this alternative method is that it can be used to bring the time gain differences within the acceptable range for the correlation technique.

To detect possible unwanted frame drops (measurement gaps), the time difference development between the video and the physiological signals was determined, with the result shown in Figure 7.

The data window used was 100 clock intervals, which corresponds to $55.9 \mathrm{sec}$ (code generation frequency $=1.79 \mathrm{~Hz}$ ). The correlation function step resolution was 0.1 clock interval $($ or $0.1 / 1.79=0.0559 \mathrm{sec})$. The calculation window was moved in steps of $1 \mathrm{sec}$ over the complete measurement. The expected linear relation is present. The small jump, occurring at one third of the measurement, coincides with a change in the experimental procedure: The first experimental run ended, and the cabin lights were turned on to allow the pilots to complete some questionnaires. Some PRN data points, as derived from the video information, were therefore found to be incorrect. Especially, the on-off times showed deviations (the on times were too large).

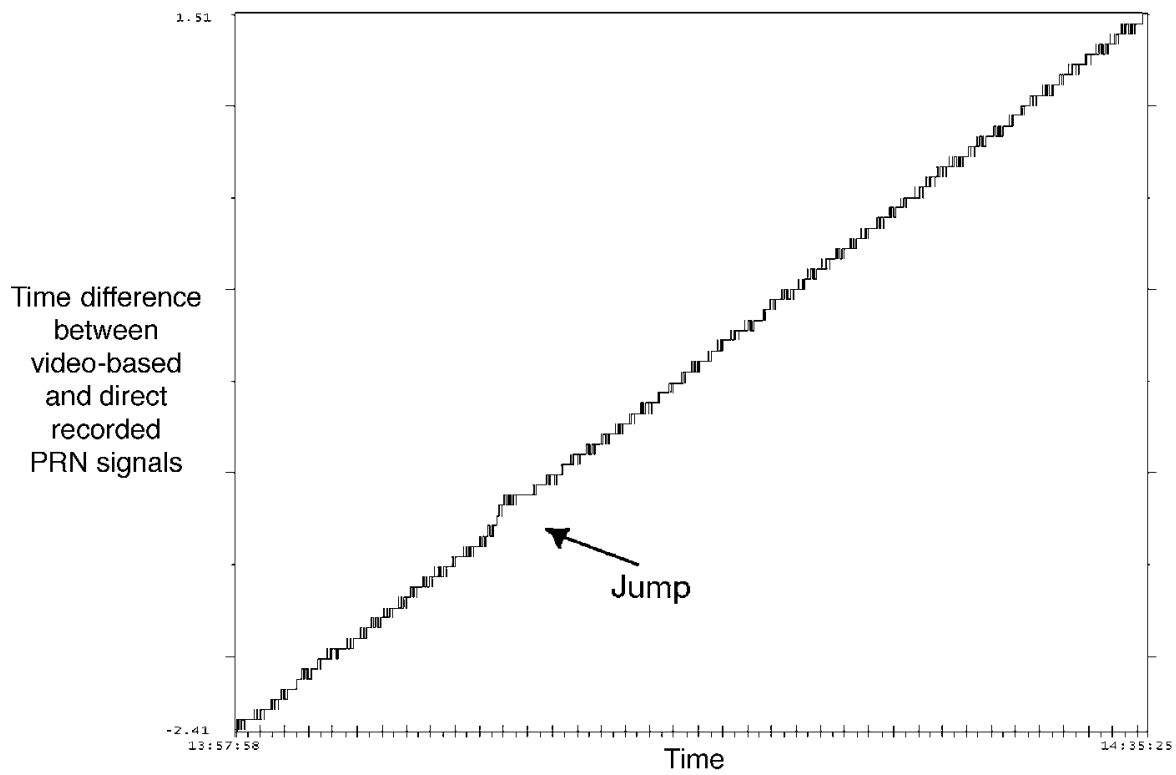

Figure 7. Time difference development for an example video. A moving window is used for the determination of the time difference. The vertical step size is caused by the (selected) resolution of the time determination algorithm. The deviation from the linear line ("Jump") is caused by a drastic change in illumination of the environment affecting the light-emitting diode on/off detection. PRN, pseudorandom noise. 
The vertical step size of the line is caused by the temporal resolution of the correlation step (set at 0.1 clock interval) in combination with some remaining noise. Therefore, the determined peak location alternated between the two resolution values surrounding the actual time difference. A smaller correlation step size or an alternative peak estimation method would reduce the vertical step size at the cost of increasing the computation times. Also note that the estimate of the time gain and the time offset between the measurements can be improved by taking the best fit of a line through all the data points.

\section{Conclusions}

This article presents an approach by which to determine time offsets between different data sets with high accuracy. The method is based on the use of a correlation function in combination with a binary PRN signal. Related signals are also used in equipment such as satellite navigation systems (e.g., GPS) and telephone communication services (GSM). The time difference determination method has been successfully applied by NLR in research projects in various circumstances. The technique is a likely candidate to be implemented in future Noldus products (Noldus, Trienes, Hendriksen, Jansen, \& Jansen, 2000).

Even though the technique is powerful, it was found that the robustness of the correlation function for larger differences in recording speed (time gain difference larger than approximately $1 \%$ ) needs improvement. Therefore, in those cases, alternative time gain difference estimations have to be used to increase the reliability of the proposed synchronization technique. Fortunately, the PRN signal provides this information in the form of observable signal level changes. Experience indicates that the observed time gain difference is normally less than approximately $0.3 \%$, and so the sensitivity to the time gain differences of the method is adequate for practical applications.

So far it has not been necessary to use the details of the PRN code: The signal waveform is totally deterministic and can be generated afterward. Correlating the measured signal with the expected code sequence is an excellent method for reducing measurement errors and resampling noise. In that reuse, the technique discussed and illustrated has sufficient growth potential for future challenges in the synchronization of valuable but hard-to-record data.

\section{REFERENCES}

ANony mous(1997).Navstar GPS Space Segment/Navigation User Interfaces (ICD-GPS-200, Revision C, Initial Release). GPS NAVSTAR JPO. See http://gps.losangeles.af.mil.

Brown, R, \& HWANG, P. (1992). Introduction to random signals and applied Kalman filtering. New York: Wiley.

Dixon, R. C. (1994). Spread spectrum systems with commercial applications (3rd ed.). New York: Wiley.

Geuze, R. H., \& HunNius, S. (2002). Measurement and analysis of eye movement and heart rate as markers of visual attention in babies. In Measuring Behavior 2002, 4th International Conference on Methods and Techniques in Behavioral Research (Amsterdam, The Netherlands, 27-30 August 2002, pp. 82-83). See http://www.noldus.com/ events/mb2002.

GoLD, R. (1967). Optimal binary sequences for spread-spectrum multiplexing. IEEE Transactions on Information Theory, IT-13, 619-621.

GoLD, R. (1968). Maximal recursive sequences with 3-valued recursive cross-correlation functions. IEEE Transactions on Information Theory, IT-14, 154-156.

Harlaar, J., Redmeijer, R. A., Tump, P., Peters, R, \& Hautus, E. (2000). The SYBAR system: Integrated recording and display of video, EMG, and force plate data. Behavior Research Methods, Instruments, \& Computers, 32, 11-16.

Hoogeboom, P. J. (2000). Human factors evaluations, data analysis and reduction techniques (HEART). In Proceedings of the 2nd IEEE Benelux Signal Processing Symposium (SPS-2000). Brussels: IEEE.

LIN, D. M., \& TsUI, J. B. Y. (2000). Comparison of acquisition methods for software GPS receiver. In ION GPS 2000 Conference.

Nibbelke, R, Ferro, D., \& Hoogeboom, P. J. (2001). Design and evaluation with the human in mind. In Air \& Space Europe: Proceedings of the Aeronautics Days 2001, Hamburg. Amsterdam: Elsevier.

NoLDUs, L. P. J. J. (1999). Visual Lab: Integrated measurement, analysis and visualization of behavior and physiological signals. In Senter Project BTS-99013 (April 1999-March 2002). Available at http://www. noldus.com/events/mb2000/program/sig_visuallab.html.

Noldus, L. P. J. J., Trienes, R. J. H., Hendriksen, A. H. M., Jansen, H., \& JANSEN, R. G. (2000). The Observer Video-Pro: New software for the collection, management, and presentation of time-structured data from videotapes and digital media files. Behavior Research Methods, Instruments, \& Computers, 32, 197-206.

Pursley, M. B., \& Roefs, H. F. A. (1979). Numerical evaluation of correlation parameters for optimal phases of binary shift registers. IEEE Transactions on Communications, Com-27, 1597-1604.

SteIn, S. R., \& VIG, J. R. (1992). Communications frequency standards. In F. E. Froehlich \& A. Kent (Eds.), The Froelich/Kent encyclopedia of telecommunications (Vol. 3, pp. 445-500). New York: Marcel Dekker.

VIG, J. R. (2001). Introduction to quartz frequency standards. Available at http://www.qct.nl/theory/toc.htm.

(Manuscript received September 5, 2002; revision accepted for publication March 29, 2003.) 